\title{
¿REDUCE, COMPLETA O ELIMINA? SOBRE EL ESTATUS DEL PROGRAMA MINIMALISTA EN LA GRAMÁTICA GENERATIVA*
}

\author{
VÍCTOR M. LONGA \\ Universidade de Santiago de Compostela
}

\author{
GUILLERMO LORENZO \\ Universidad de Oviedo
}

\section{RESUMEN}

El Programa Minimalista es una propuesta teórica sustantiva con relación a las cuestiones tradicionalmente planteadas por la Gramática Generativa: ¿Qué es el conocimiento del lenguaje? ¿Cómo está representado en la mente/cerebro de los hablantes? ¿Cómo se desarrolla en la mente infantil? ¿Cómo ha evolucionado en la especie? Las respuestas a estas preguntas son tan diferentes a las de modelos generativistas previos que no es posible seguir sosteniendo, como viene siendo habitual, que el Programa Minimalista es un desarrollo natural u orgánico de la Teoría de Principios y Parámetros. En este artículo discutimos dos diferentes versiones de este supuesto y concluimos que el minimalismo solo tiene verdadero sentido como un programa de investigación eliminativista.

Palabras clave: Gramática Generativa, minimalismo, adquisición, reduccionismo, eliminativismo.

\section{ABSTRACT}

The Minimalist Program is a substantive theoretical proposal concerning the traditional questions brought to the fore by Generative Grammar: What is knowledge of language? How is it represented in the mind/brain of speakers? How does it grow in the mind of children? How did it evolve in the species? The answers to these questions are so different from that of previous generative models that the customary contention that the Minimalist Program is a natural or organic development of the Theory of Principles and Parameters cannot be held anymore. In this article we discuss two different variants of such a contention and we conclude that minimalism only makes sense as an eliminative program.

Key Words: Generative Grammar, minimalism, acquisition, reductionism, eliminativism.

* Este trabajo ha sido realizado al amparo del Proyecto de Investigación Biolingüística: evolución, desarrollo y fósiles del lenguaje (FFI2010-14955/FILO), subvencionado por el Ministerio de Ciencia e Innovación y cofinanciado parcialmente por fondos FEDER. Queremos agradecer las muy valiosas sugerencias y comentarios de dos revisores anónimos de la Revista Española de Lingüistica. 


\section{INTRODUCCIÓN}

El Programa Minimalista (desde ahora, PM) ha propiciado una nueva manera de conceptualizar la Facultad del Lenguaje (en adelante, FL). No es fácil decidir, sin embargo, cuál es la naturaleza exacta de la relación de esta nueva perspectiva conceptual con los modelos generativos anteriores. Los casi dos decenios de puesta en práctica del programa no han arrojado mucha luz en este sentido; al contrario, la situación resulta bastante más confusa en la actualidad que en el propio arranque del minimalismo (Chomsky 1993; 1995a). Nos encontramos, en primer lugar, con reductos del generativismo en que el PM ha tenido una influencia casi nula, como es el caso de la «teoría de la adquisición» (Longa y Lorenzo 2008). Apreciamos, en segundo lugar, que una mayoría de los practicantes del minimalismo lo interpretan como una continuación natural del Modelo GB (Chomsky 1981) que en nada cuestiona las conclusiones generales de aquel modelo ni de la «Teoría de Principios y Parámetros» (desde ahora, TPP; Chomsky y Lasnik 1993) que lo ampara. Dentro de esta línea general observamos, además, una visión tendente a considerar el minimalismo como un programa de reducción de principios explicativos, cuya corrección ha sido previamente demostrada por GB, a un vocabulario más básico y que además permite generalizaciones de más amplio alcance, no localizadas en el ámbito de lo lingüístico (Hornstein et al. 2005). Observamos, por otra parte, una cierta inclinación a considerar que el PM y el Modelo GB se completan mutuamente (o convergen), en el sentido de que cada uno de ellos capta aspectos coexistentes y que deben formar igualmente parte de una modelización teórica adecuada de la FL (Chomsky 2007; 2009). Ambas tendencias tienen en común que dan al PM un papel positivo en la construcción de la TPP, pasando por alto el potencial eliminativista del primero sobre los supuestos básicos de la segunda, puesto en cambio de relieve por otros autores (Lorenzo y Longa 2009; 2011). Se trata realmente de una situación muy confusa, a la que sin duda ha contribuido en buena medida la indefinición del propio Chomsky, quien ha oscilado entre una interpretación eliminativista fuerte (Chomsky 2000a; 2004) y una metodológica más suavizada y conciliadora del minimalismo con la teoría gramatical inmediatamente anterior (Chomsky 2007; 2009).

La tesis principal que queremos defender en este trabajo se resume en pocas palabras: en la medida en que se plantee como una aportación con un contenido teórico sustantivo y no como un mero programa de aplicación de pautas metateóricas de alcance general, el minimalismo solo puede ser eliminativista. La razón es simple: el PM es una teoría asociada a un conjunto de postulados falsables sobre la naturaleza de la FL claramente confrontados con los que definen no ya al mode- 
lo GB, sino a la propia TPP. Un aspecto crucial de nuestro argumento es que, entre tales postulados, están centralmente implicados varios relacionados con el desarrollo individual del lenguaje, lo que significa que el PM no es una teoría en modo alguno ajena al Problema de Platón. Esta observación es decisiva, porque tanto la posición reduccionista como la convergentista descansan en el supuesto de que el minimalismo es ajeno a la cuestión de la adquisición y que se aplica una vez resuelto (reduccionismo) o paralelamente a la resolución del Problema de Platón (convergentismo). Desde el punto de vista que defendemos aquí, en cambio, el PM plantea una respuesta característica al problema de la adquisición del lenguaje, cuya confirmación (incluso parcial) desmiente los postulados de la TPP. Por tanto, no cabe una interpretación reduccionista ni convergentista del minimalismo, salvo que el PM sea falso.

El desarrollo de nuestro argumento presupone un cierto conocimiento de los supuestos fundamentales de la TPP y del PM. Nosotros los hemos sintetizado en dieciséis puntos críticos que, sin agotar la caracterización de ambos modelos, son suficientes para captar la manifiesta brecha teórica existente entre ellos. Hemos además decidido presentarlos en un Apéndice al final del trabajo (Ap.), pensando en que los lectores ya familiarizados con ellos no se vean obligados a leer una vez más lo consabido. Al resto de los lectores les recomendamos comenzar el artículo por esa sección. A lo largo del texto insertamos además referencias a los epígrafes del apéndice que consideramos más relevantes en cada punto de la discusión. El resto del trabajo se organiza así: la sección 2 trata la distinción entre minimalismo metodológico y minimalismo ontológico, intentando dejar claro que el primero siempre ha estado presente en el desarrollo de la Gramática Generativa y que el PM solo puede caracterizarse como una concreción del segundo. La sección 3 explica la propuesta reduccionista que, de acuerdo con numerosos autores, se corresponde con la «visión de consenso» sobre el papel del minimalismo en el desarrollo de la Gramática Generativa; se ocupará asimismo de explicar por qué disentimos de ese consenso. Por último, la 4 se ocupa de la posición más recientemente defendida por Chomsky, quien señala que el minimalismo y el maximalismo (cf. Ap.1) son facetas complementarias (respectivamente, ascendente y descendente) de un único programa de investigación que, idealmente, debe dar lugar a resultados convergentes. Explicaremos qué significa todo ello y, sobre todo, por qué se trata de un modelo inviable tal cual lo plantea Chomsky. En la sección de conclusiones insistiremos en el carácter necesariamente eliminativista del minimalismo y en que podemos encararlo sin sensación alguna de traición a las tres décadas precedentes de desarrollo de la visión generativa sobre el lenguaje. 


\section{Minimalismo Metodológico y Minimalismo ONTOLÓGICO}

Chomsky 2000a, p. 73; 2002a, p. 96 y ss.; y Martin y Uriagereka 2000, p. 1, han aclarado la diferencia entre un minimalismo metodológico y otro ontológico. El primero, muy lejos de ser una aportación específica del PM, hace referencia a un ideal que guía las descripciones científicas relativas a cualquier tipo de fenómeno (también la FL). Un viejo conocido de cualquier científico, por tanto, pues no se trata de otra cosa que de la propiedad de «parsimonia» o «navaja de Occam», acaso el primer principio del libro de estilo que rige la elaboración de buenas teorías y la práctica científica en general. En palabras de Wilson 1998, p. 291:

Cuantas menos unidades y procesos se utilicen para explicar el fenómeno, mejor. Debido al éxito de la parsimonia en las ciencias físicas, en la actualidad no necesitamos una sustancia imaginaria denominada flogisto para explicar la combustión de la leña, o un éter inexistente que llene el vacío del espacio.

Así, el minimalismo metodológico se caracteriza por aplicar en el nivel de la teoría (importante: no en el del objeto de estudio) la navaja de Occam, buscando la mayor simplicidad posible en el vocabulario que la teoría en cuestión relaciona con los mecanismos y principios activos en un determinado dominio de la experiencia. Se eliminará, atendiendo a esta pauta, todo lo que pueda derivarse de algo independientemente motivado en el marco de esa teoría o de teorías mejor establecidas. Este tipo de minimalismo dicta, por tanto, que no debemos introducir nuevos principios en nuestras explicaciones si ya contamos con otros, justificados independientemente, capaces de cumplir la misma función.

El minimalismo metodológico siempre ha estado presente en la Gramática Generativa (cf. Chomsky 1998a sobre su importancia). A su aplicación se debe, por ejemplo, la formulación de reglas como A-sobre-A o las restricciones de isla de Ross 1967, la abolición del orden extrínseco de las reglas o las condiciones sobre las transformaciones de Chomsky 1973 (cf. Longa 1999a y Van Riemsdijk y Williams 1986). Pero, como señalan Martin y Uriagereka 2000, p. 1, este minimalismo no se corresponde con una verdadera tesis sobre la naturaleza de la FL, salvo en un sentido débil: «[it] is just a new way to refer to what many people have been doing for a long time: seeking the best way to theorize about a particular domain of inquiry». Dicho de otro modo, el minimalismo metodológico se corresponde con las pautas de adecuación internas a las teorías, no con la naturaleza última de los fenómenos sometidos a escrutinio teórico.

Bien diferente es lo que plantea en cambio el minimalismo ontológico, el cual implica la introducción de una «tesis fuerte» (Martin y Uriagereka 2000, p. 1) nunca antes considerada en Gramática Generativa. 
Tal tesis no se plantea al respecto de la parsimonia o la adecuación interna de la teoría sobre la FL sino, directamente, al respecto de si la propia FL manifiesta o no una propiedad equivalente (Chomsky 1998a, p. 120). En otros términos, el minimalismo ontológico se plantea la cuestión de hasta qué punto el lenguaje (no la teoría sobre el lenguaje) puede considerarse bien diseñado. En palabras de Chomsky 2000a, pp. 72-73:

[...] es posible considerar algunas preguntas nuevas sobre FL. En particular, podemos preguntar: ¿en qué medida está bien diseñada FL? Supongamos que a un super-ingeniero se le hubieran dado especificaciones de diseño para el lenguaje: «Aquí están las condiciones que FL debe satisfacer: tu tarea es diseñar un mecanismo que satisfaga estas condiciones de alguna manera óptima [...] La pregunta es: ¿cuánto se acerca el lenguaje a tal diseño óptimo?».

La propuesta de partida (susceptible de corrección empírica, no por razones de adecuación teórica interna) es la llamada «Tesis Minimalista Fuerte», que «explora la posibilidad de que el lenguaje se aproxime al buen diseño en este sentido» (Chomsky 2000a, p. 76) y afirma que «el lenguaje es una solución óptima a las condiciones de legibilidad» planteadas por los sistemas externos o de actuación (senso-motrices y de pensamiento). En la medida en que la tesis no se vea refutada empíricamente, podrá sostenerse que la FL manifiesta un diseño que «no ofrece más maquinaria de la necesitada para satisfacer las exigencias mínimas de legibilidad y [...] funciona de la manera más simple posible» (Chomsky 2000a, p. 94).

Todo lo anterior implica que el PM atribuye a la FL propiedades muy diferentes a las que le eran atribuidas en modelos anteriores, basados en un rico vocabulario de elementos y principios específicos de dominio (es decir, puramente gramaticales) [cf. Ap. 1-7]. Todo este vocabulario, sin embargo, se ha visto desplazado en el minimalismo por otro basado directamente en los principios independientemente atribuidos a los sistemas cognitivos externos, pero en contacto directo con la FL (Hauser et al. 2002), o en categorías que se derivan por necesidad conceptual y se supone por ello que surgen de modo espontáneo (sin ninguna instrucción o directriz específica) y tienen un rango de actuación no limitado a ningún dominio en concreto (lenguaje, cognición, mundo orgánico o materia inorgánica). No se trata, por tanto, de una simple cuestión de limar redundancias o eliminar elementos superfluos en las descripciones. El minimalismo ontológico apunta directamente a la naturaleza, organización y origen del lenguaje, en clara confrontación con cualquier modelización teórica planteada antes sobre cualquiera de esos extremos. Esto no quiere decir que el PM no asuma el principio de parsimonia de la práctica científica normal (lo hace, sin duda) o que no incorpore otras pautas metodológicas características. Al contrario, incorpora al menos una especialmente distintiva e importante: la de explorar en primer lu- 
gar la Tesis Minimalista Fuerte y solo aceptar que la FL se aparta de ella si media desmentido empírico. Nótese que tal metodología no hace más que confirmar que el PM se corresponde con un contenido teórico sustantivo propio y diferente al de otros modelos generativos.

En resumen, cuando el minimalismo ontológico apela a la máxima «reduce computational complexity» (Martin y Uriagereka 2000, p. 24) no alude a la aplicación, por otra parte rutinaria, de la navaja de Occam; se refiere, en un sentido mucho más profundo, a una visión particular sobre la manera de ser de la FL, basada en el rechazo al tipo de sistema autocontenido, muy complejo y altamente especializado que la Gramática Generativa defendió sin ambigüedad durante algo más tres décadas.

\section{La Visión de consenso sobre el Programa Minimalista}

Son muchos los autores que entienden que el PM consiste en una extensión del Modelo GB y es por tanto un desarrollo en un sentido particular de la TPP (entre otros, Boeckx 2006a; 2006b; Eguren y Fernández Soriano 2004; Hornstein 2001; 2009; Hornstein et al. 2005; Freidin y Vergnaud 2001; Friedemann y Rizzi 2000; Kitahara 2006; Rizzi 2004 y Valois 2000). En palabras muy representativas, de Rizzi 2004, p. 335: «the Minimalist Program represents, I believe, a development in full continuity with the principles and parameters guidelines».

Este planteamiento se basa en la consideración de que la TPP es una solución acertada y definitiva al Problema de Platón, cuestión en torno a la cual existe un amplio consenso entre los generativistas, lo que hace que el formato del Modelo GB se sienta como adecuado con relación a tal problema [Ap. 15]. Esto no obsta, sin embargo, para que dicho formato pueda verse sometido a la aplicación de criterios de simplicidad, elegancia o naturalidad que, lejos de cuestionarlo, se aplican bajo el supuesto de su corrección empírica. Esta visión de consenso se hace especialmente explícita en Hornstein et al. 2005, pp. 5, 59, donde recibe por primera vez tal nombre. Expresado en términos operativos, el planteamiento supone someter a la cirugía del minimalismo metodológico las conclusiones esencialmente correctas del Modelo GB con relación al problema de la adquisición (cf. además las formulaciones de la misma idea en Boeckx 2006a, p. 13; Freidin y Vergnaud 2001, pp. 642-643 y Kitahara 2006, p. 5). Estos fragmentos de Hornstein 2009 resumen perfectamente la visión de consenso:

On the assumption that GB gives a decent first approximation of what the laws of grammar are (i.e. provides a reasonable description of FL), this translates into a research program that aims to derive GB from simpler, more natural assumptions. If this is correct, then the Minimalist Program should not be understood as replacing GB but as presupposing its general validity (pp. 115-116). 
[...] the Minimalist Program (MP) is a continuation of the GB research program, not a competitor. First, MP starts from the assumption that GB is roughly correct. It accepts both the general problems identified for solution (e.g. Plato's Problem) and the generalizations (laws) that have been uncovered (at least to a good first approximation). The second way that MP continues the GB program is in its identification with the Rationalist research strategy that sits at the core of Chomskyan enterprise in general and GB in particular. As is well known, Rationalists, in contrast with Empiricists, endorse the postulate that the mind/brain is endogenously highly structured $[\ldots]$ (p. 178).

Obsérvese que, según esta perspectiva, el PM sería un planteamiento ontológicamente vacío, en términos de la distinción establecida en el apartado anterior. Dentro de su característica posición oscilante, Chomsky se acerca en alguna ocasión a esta interpretación del PM, como cuando escribe:

the basic contours of an answer to the problems of explanatory adequacy [i.e. Problema de Platón; VML y GL] might be visible. On that tentative assumption, we can turn more seriously to the why questions that transcend explanatory adequacy. The minimalist program thus arose in a natural way from the successes of the P\&P approach (Chomsky 2009, p. 25).

Se trata de una interpretación que choca radicalmente con la expuesta por el propio Chomsky en otros lugares (cierto que en textos anteriores), donde razona que el PM corrige y elimina el trabajo precedente formulado en términos del Modelo GB (no por ello inútil) o, más sutilmente, que la fortaleza de las conclusiones alcanzadas por este último modelo cuestionan que el PM pueda llegar a revelarse empíricamente correcto (nuestras aclaraciones entre corchetes):

Todo lo explicado en esos términos [los de GB; VML y GL] se ha descrito mal, debiendo entenderse más bien en términos de condiciones de legibilidad en el interfaz [...] (Chomsky 2000a, p. 95).

Una asunción básica del trabajo en el marco P\&P, con sus importantes logros, es que todo lo sugerido [por el PM; alude a la perfección del lenguaje; VML y GL] es falso: que el lenguaje es altamente imperfecto (Chomsky 2000a, p. 95; cf. también Chomsky 1998a, p. 120).

No hay nada semejante a la estructura profunda y la superficial en el sentido técnico del término, y todo lo que se haya explicado en esos términos, a esos niveles, se comprendió de mala manera y describió de manera aún peor (Chomsky 2000b, p. 38).

[...] no hay rección, no hay rección propia, no hay una teoría del ligamiento inherente al lenguaje, ni hay interacciones de otra clase. En la medida en que el lenguaje sea perfecto, es necesario prescindir de todo esto (Chomsky 2000b, p. 39). 
El supuesto esencial de las obras más altamente productivas de los últimos tiempos -y de sus logros, sin duda impresionantes-, es que todo lo que acabo de decir [sobre el PM; VML y GL] es un craso error, que los lenguajes son sumamente imperfectos en todos estos sentidos, como cabría esperar; poseen índices y niveles de barra, estructuras $\mathrm{P}$ y estructuras $\mathrm{S}$, toda clase de relaciones, etc., etc. Por eso insisto en que no es tarea fácil empeñarse en mostrar lo contrario. A pesar de todo, creo que lo contrario bien pudiera ser verdad (Chomsky 2000b, p. 40).

Compartimos las opiniones expresadas por Chomsky en esta última serie de observaciones y no es difícil explicar por qué nos parecen correctas. La visión de consenso se asienta en la idea de que cada una de las conclusiones alcanzadas por la TPP en respuesta a los desafíos del Problema de Platón resultan reformulables a través de un vocabulario alternativo que las compacta o simplifica sin corregirlas. Llamemos $\mathrm{X}_{\mathrm{GB}}$ a una cualquiera de esas conclusiones e $Y_{P M}$ a la reformulación que resulta de aplicar sobre ella la cirugía del minimalismo metodológico. Según la visión de consenso, $\mathrm{Y}_{\mathrm{PM}}$ es una formulación más sencilla, natural o elegante que $\mathrm{X}_{\mathrm{GB}}$ porque analiza la ley expresada por ambas formulaciones en términos de un vocabulario proyectable más allá del ámbito lingüístico que, en virtud de la navaja de Occam, nos lleva a preferirlo sobre el vocabulario de la formulación original. Sea $\mathrm{X}_{\mathrm{GB}}$, p. ej., la ley sobre la estructura de frase que establece que un núcleo de una determinada categoría complementada opcionalmente por la proyección máxima de otra categoría compone una proyección intermedia con la categoría del núcleo; y sea $\mathrm{Y}_{\mathrm{PM}}$ la reducción de esa ley al principio «combinación binaria + proyección asimétrica». De acuerdo con la visión de consenso, la relación entre una y otra formulación sería del tipo que se expresa en (1), donde $\approx$ representa el tipo de igualdad propio de las leyes puente en las fórmulas que expresan la reducción de unos aparatos teóricos a otros más básicos (cf. Fodor 1975, pp. 32-34):

\section{(1) $\mathrm{X}_{\mathrm{GB}} \approx \mathrm{Y}_{\mathrm{PM}}$}

Es cierto que este tipo de reducciones se aplican de ordinario entre las leyes de una determinada ciencia y las de otra que se considera más básica (por ejemplo, la reducción de buena parte de la química a leyes de la física). Sin embargo, seguimos teniendo exactamente una relación de reducción, si lo que aparece a izquierda y derecha en la ley puente continúan siendo leyes de una misma disciplina, aunque apareciendo ahora en la parte derecha de la formulación una misma ley que hace uso de un vocabulario generalizable (actual o potencialmente) a las leyes propias de otros dominios de investigación, tal como se le supone al vocabulario minimalista. 
Ahora bien, lo que no puede cumplirse en ningún caso en los procesos de reducción teórica de este tipo es que las formulaciones que aparecen a uno y otro lado de la ley puente sean mutuamente contradictorias. En tal caso, la nueva formulación no reduce (simplifica, naturaliza o dota de mayor elegancia) a la precedente, sino que directamente la elimina. Y esto es lo que sucede en la relación entre las leyes formuladas en términos del Modelo GB y las formuladas en términos del PM, cuya carga teórica e implicaciones empíricas son radicalmente diferentes, como se puede comprobar revisando los dieciséis puntos de AP. En esta sección, para subrayarlo, nos limitamos a elaborar algo más el ejemplo propuesto arriba al respecto de las teorías pre-minimalista y minimalista sobre la estructura de frase.

Nos centraremos en la consideración por parte de autores como Rizzi 2000 o Eisenbeiß 2009 del estatus de la «teoría desnuda de la estructura sintagmática» (Chomsky 1995b), que reemplazó a la configuración X-barra del Modelo GB. Eisenbeiß 2009, p. 287, no concede relevancia ontológica alguna a tal sustitución: «the X-bar principles are replaced by a more general principle which requires elements that occupy a high position in the syntactic representation to precede elements which are positioned lower in the syntactic tree». Lo que plantea Rizzi 2000, p. 284, es algo muy similar. En un comentario sobre la rica estructura de la periferia izquierda, señala:

In recent years, detailed studies on the clause and its left periphery are bringing to light a very complex cartography, which is nevertheless very simple in that its atomic component is always the same, the basic X-bar schema (or the even more rudimentary structural schemata assumed in Kayne 1994 and Chomsky 1995).

Como se aprecia, ni Eisenbeiß ni Rizzi otorgan relevancia ontológica a la teoría desnuda, que es, a su juicio, respectivamente «un principio más general»o «un esquema estructural más rudimentario» que la X-barra. El estatus de esas dos alternativas sería el de meras variantes notacionales para dar cuenta del mismo fenómeno. Esto deja de lado, sin embargo, las enormes implicaciones ontológicas de la supresión del esquema X-barra por la teoría desnuda y la muy diferente arquitectura asumida para la FL (cf. Longa 2008 y Lorenzo y Longa 2009 para más detalles). La X-barra efectuaba la predicción de que el conocimiento de la estructuración jerárquica de las frases estaba pre-especificado en el genotipo lingüístico, esto es, "encoded in the genes of the children» (Smith 1999, p. 173). De ese modo, la X-barra se tomaba como «part of the basic blueprint of language» (Hyams 2002, p. 229). Y en el esquema estaban implicados varios principios específicos de dominio: endocentricidad (toda estructura debe tener un núcleo), proyección categorial 
(el núcleo se proyecta en dos niveles) o minimalidad y maximalidad categorial (una proyección implica un núcleo y viceversa).

Sin embargo, según el PM la X-barra, como tantos otros mecanismos específicos del lenguaje, se planteó desde una visión en la que la FL «se comprendió de mala manera y describió de manera aún peor» (Chomsky 2000b, p. 38). La teoría desnuda, basada en razones de necesidad conceptual que surgen espontáneamente, elimina todo el aparato específico de dominio propio de la X-barra y, crucialmente, la asunción de que el origen de la estructuración jerárquica está en los genes. La teoría desnuda postula una única operación, «Ensamble», aplicada recursivamente, que fusiona dos objetos $\{\mathrm{A}, \mathrm{B}\}$ en un nuevo objeto $\mathrm{C}$. Pero debe notarse que las condiciones de esa fusión son las más sencillas de entre todas las posibles o concebibles desde una perspectiva conceptual o lógica (cf. Chomsky 1995b, p. 396); el ensamble es:

1) binario (en vez de ternario, cuaternario, etc.),

2) asimétrico (la etiqueta de la estructura resultante siempre se identifica con uno de los elementos fusionados, en lugar de con los dos a la vez, con uno intermedio o con un tercer elemento),

3) de aplicación ilimitada (no hace falta estipular límite alguno) y

4) preservador de la estructura (en lugar de eliminar o alterar parte de la estructura o de alterar las relaciones jerárquicas).

Son propiedades que, en algunos casos, se han revelado igualmente activas en dominios inesperados desde una perspectiva exclusivamente lingüística. Véase, p. ej., Cherniak 2005; 2009, sobre el modo de organización binario en las redes de combinación neuronal, que este autor no remite a ningún tipo de instrucción genética, sino a la operatividad espontánea de criterios de optimidad en la organización.

De modo semejante, tanto la operación Ensamble en sí misma, como las condiciones formales que satisface, son muy sencillas, las más sencillas posibles. Por ello, la teoría desnuda asume que no es necesaria ninguna especificación especial para dar cuenta de ellas: ni ubicarlas en el plano genético, ni concederles estatus de elementos específicos de dominio (es decir, gramaticales), sino que la operación y sus condiciones surgen por defecto, a modo de principios de auto-organización: «Phrase structure theory is essentially given on grounds of virtual conceptual necessity» (Chomsky 1995b, p. 403). Se rechaza, pues, apelar a ningún tipo de especificación puramente gramatical: Ensamble y sus propiedades distintivas son condiciones muy simples y genéricas, que surgen de modo espontáneo en ausencia de especificaciones en otro sentido y para las que no es necesario establecer ninguna estipulación especial. 
Con lo dicho se puede entender que la explicación de la estructuración jerárquica mediante la X-barra o mediante la teoría desnuda no se pueden considerar, como hacen Eisenbeiß o Rizzi, meras variantes notacionales, sino dos propuestas confrontadas. Ambos autores, al plantear que X-barra y teoría desnuda son simples variantes que hablan de lo mismo, pero con diferente vocabulario, pasan por alto la cuestión de si el niño necesita y dispone de un conocimiento a priori de la estructura, y esa es la clave de gran parte de las diferencias entre el Modelo GB y el PM. La visión de consenso asume que la cuestión ya ha sido respondida satisfactoriamente por el primero (el niño necesita y tiene a su alcance una pauta innata sobre la forma y estructura de las frases) e ignora que el PM plantea una respuesta alternativa (la forma y estructura de las frases emergen espontáneamente y no requieren de instrucciones genéticas para ser adquiridas y usadas) [cf. Ap. 7-15]. Por esta razón, nos parece un error basar la interpretación de la relación entre uno y otro planteamiento en el tipo de leyes puente que hemos simbolizado en (1); el PM no reduce, sino que elimina el Modelo GB.

\section{LAS ESTRATEGIAS «DESCENDENTE»Y «ASCENDENTE» DE LA GRAMÁTICA GENERATIVA}

Lo dicho en la sección anterior implica que la visión de consenso no reconoce al PM la capacidad de dar respuestas sustantivas al Problema de Platón. No lo hemos destacado allí con más energía porque se trata de una cuestión que, a nuestro juicio, se cumple de manera aún más acusada, y acaso inesperada, en la interpretación del papel del PM en el desarrollo del generativismo planteada por Chomsky en algunos trabajos recientes, de la que vamos a ocuparnos ahora.

Según esta interpretación, el PM es un proyecto complementario a los anteriormente desarrollados por el generativismo (por tanto, también al Modelo GB), aunque en un sentido sutilmente diferente al planteado por la visión de consenso. De acuerdo con Chomsky, los modelos no minimalistas (desde Aspectos al Modelo GB) han enfocado la investigación sobre la FL adoptando una perspectiva descendente, que básicamente consiste en situar en el primer plano la resolución del Problema de Platón. En palabras del propio Chomsky 2007, p. 4: «Throughout the modern history of generative grammar, the problem of determining the character of FL has been approached from top-down: How much must be attributed to UG to account for language acquisition?» (en Chomsky 2009, p. 25, el autor se expresa idénticamente). La aplicación de esta perspectiva da lugar a una visión según la cual «FL must be rich, highly structured, and substantially unique» (Chomsky 2007, p. 2; cf. también 
Chomsky 2009, p. 20), lo que se traduce en la postulación de una Gramática Universal (desde ahora, GU) conformada por principios altamente específicos de dominio remitidos al plano genético: es decir, a la postulación de un genotipo lingüístico (Chomsky 1980).

Complementariamente, el PM se basaría, de acuerdo con esta interpretación, en la aplicación de una perspectiva ascendente, que Chomsky 2007, p. 4, caracteriza diciendo que el minimalismo «seeks to approach the problem from bottom-up: How little can be attributed to UG while still accounting for the variety of I-languages attained, relying on third factor principles?» (cf. también Chomsky 2009, p. 25, así como Boeckx 2006a, p. 95). También de acuerdo con Chomsky 2007, p. 26: «The two approaches should, of course, converge, and should interact in the course of pursuing a common goal»; e insiste en la misma idea cuando afirma que «insofar as the two lines of inquiry converge, they clarify the questions that have been at the heart of theoretical study of language since its origins: to determine the basic properties of FL» (cf. también Chomsky 2009, p. 25).

La principal diferencia de esta interpretación con relación a la de la visión de consenso es que, según la de Chomsky, las dos perspectivas pueden aplicarse simultáneamente, según los intereses y la dedicación particular de cada investigador; para la visión de consenso, en cambio, el minimalismo presupone la previa resolución del Problema de Platón y ser aplicado sobre los resultados que va arrojando la teoría de la adquisición. En términos más próximos a lo que sería la aplicación del método chomskyano, el adquisicionista identifica todos los aspectos del conocimiento del lenguaje que no pueden derivarse de la experiencia; el minimalista, en paralelo, identifica todos aquellos aspectos del conocimiento del lenguaje que pueden derivarse de las propiedades de los sistemas externos con que el lenguaje establece relaciones de interfaz o de principios de alcance general sobre el procesamiento de datos, auto-organización de la forma, gestión de la complejidad, etc. (es decir, principios de tercer factor [Ap. 10]). A continuación, aquellos aspectos del lenguaje así minimizados que coincidan con los identificados por el adquisicionista como no aprendibles (no adquiribles mediante un proceso inductivo o guiado por los datos) se considerarán explicados; el resto, se remitirá al genotipo lingüístico.

No pretendemos criticar la bondad del método. Al contrario, opinamos que se trata de un método que merece ser operativizado y aplicado con todo rigor (Longa y Lorenzo 2008). Disentimos, sin embargo, de la opinión de que la aplicación de este método implique de algún modo mantener vivas las teorías pre-minimalistas. Lo que su aplicación rigurosa requerirá por parte del adquisicionista, como se señaló arriba, será identificar los aspectos del conocimiento del lenguaje que el niño no deriva de la experiencia. En esta fase, sin embargo, remitir al genotipo 
tales aspectos del lenguaje no se corresponde con ninguna necesidad realmente planteada por el método. Esto significa que, en este punto, el teórico descendente no necesita hacer uso de una teoría fuertemente geneticista como las que antecedieron al PM. Insistimos: tales prejuicios teóricos serían por completo irrelevantes para la consumación del método en esta fase de ejecución.

Por lo que respecta a la remisión al genotipo lingüístico de los aspectos que, por su parte, el teórico ascendente no consiga minimizar (o explicar), resulta igualmente claro que no presupone en ningún aspecto la corrección de los modelos anteriores al PM. De acuerdo con Chomsky, este tipo de corrección de la Tesis Minimalista Fuerte debería afectar idealmente a una parte muy residual del conocimiento lingüístico [veáse Ap. 11]. En tal caso, muy poco, o prácticamente nada, se mantendría en pie de la compleja arquitectura gramatical y la rica estructura de preconceptos lingüísticos propuestas por el Modelo GB con relación a la FL. Y en caso contrario, es decir, si el alcance de esas correcciones estuviese lejos de ser tan marginal como el ideal del PM plantea hipotéticamente, entonces sencillamente resultaría que el PM es falso. Las expectativas y mecanismos minimalistas están en tan fuerte oposición con los de los modelos anteriores (cf. Longa 2006, 2008; Lorenzo 2007a, 2007b; Longa y Lorenzo 2008; Lorenzo y Longa 2003, 2009), que el ideal de convergencia planteado por Chomsky resulta directamente inviable. Debe observarse, finalmente, que la aplicación rigurosa del método que plantea Chomsky, lejos de hacernos ver el minimalismo como un programa complementario y que debe aplicarse en paralelo al que por su parte se encarga de los problemas de la adquisición, lo caracteriza más bien como una teoría con una aportación sustantiva y diferenciada sobre el Problema de Platón (Longa y Lorenzo 2008).

\section{Conclusiones}

La discusión planteada en este trabajo demuestra que es difícil aceptar un tipo de relación positiva o constructiva, ya sea de tipo reductivo o convergentista, entre el Modelo GB y el PM. Tal posición es desde luego insostenible si se concede al segundo un contenido ontológico sustantivo con relación a la naturaleza de la FL, su desarrollo en el individuo, su evolución en la especie y su posición en la organización de la mente humana. Desde tal perspectiva ontológica, el lenguaje se concibe de tal manera que la única relación posible con los modelos precedentes es la de su eliminación: el PM refuta el Modelo GB (y viceversa).

Nada de ello implica que, más allá de factores sociológicos obvios, no exista una continuidad histórica entre ambos proyectos. De hecho, ciertas señas de identidad pueden considerarse comunes a los dos mo- 
delos (como el peso concedido a las propiedades de las piezas léxicas) y diferenciadoras con relación a los modelos vigentes en las décadas precedentes (centrados en reglas y condiciones sobre su aplicación). Pero, sobre todo, esa continuidad existe en la medida en que los dos tratan de dar respuesta a las mismas preguntas (en qué consiste el conocimiento del lenguaje, cómo se adquiere, cómo ha evolucionado en la especie, cuál es su base física) y desde perspectivas generales coincidentes, aunque obviamente matizadas (biologicismo, internismo, innatismo, adhesión a una visión computacional o representacional de la mente). Significa esto que el PM no es una derivación azarosa e inmotivada a partir de las encarnaciones previas de la lingüística generativa, sino un resultado al que se ha llegado mediante el cuestionamiento reflexivo de un mismo conjunto de cuestiones marco. De ahí la unidad del programa chomskiano en su totalidad. No tiene sentido, sin embargo, tratar de situar la continuidad de sus diferentes proyectos en el contenido característico de sus propuestas con relación a esas cuestiones, donde resulta en cambio evidente la brecha abierta entre ambos planteamientos. Esta discontinuidad conceptual o teórica, sin embargo, no debe extrañar ni ser rechazada por los generativistas. Se trata exactamente del tipo de rupturas que facilitan intermitentemente, en un sentido $u$ otro, los avances más significativos en cualquier dominio de investigación.

\section{APÉNDICE. \\ El Modelo GB y el Programa Minimalista: PRINCIPALES DIFERENCIAS}

Este apéndice pretende mostrar la imposibilidad de considerar que el PM es una simple extensión de GB si se toma en serio la perspectiva ontológica del minimalismo. El PM no supone simplemente reformular el contenido de la GU mediante criterios de simplicidad o parsimonia, ni implica unos cuantos cambios técnicos, o retocar la arquitectura de la FL planteada por GB, o derivar principios gramaticales de otros principios. La clave es que en el minimalismo ontológico los principios gramaticales son eliminados, y eso conduce, sencillamente, a la eliminación de la noción de GU y a cambios drásticos en la arquitectura de la FL. En resumen, todo ello produce una ontología muy diferente del objeto de estudio.

Para mostrar las muy variadas implicaciones de todo esto, adoptamos la estrategia de formular preguntas referidas a muy variados aspectos (arquitectura, diseño, asunciones biológicas subyacentes, etc.), con el objetivo de analizar cómo ambos modelos responden a cada una. El conjunto de las respuestas mostrará las grandes diferencias existentes. Por otro lado, aunque nos centraremos en GB como ejemplo paradig- 
mático de modelo no minimalista, por ser el más articulado, además de por la razón obvia de que la visión de consenso lo considera el germen directo del PM, debe tenerse en cuenta que el resto de modelos previos a GB han respondido de manera idéntica a la mayor parte de cuestiones planteadas.

\section{AP. 1. ¿Qué posición define a los dos modelos?}

Desde una perspectiva neutra, independiente de marcos concretos, el lenguaje supone un procedimiento cognitivo que permite exteriorizar nuestros pensamientos e interiorizar los pensamientos de otros. De manera equivalente, ese procedimiento relaciona sucesiones de sonidos o gestos y secuencias de sentidos. Cada una de esas dos capacidades es controlada por un sistema cognitivo concreto: la primera es controlada por el Sistema Senso-Motor o Articulatorio-Perceptivo (A-P), que rige el control motor de gestos articulatorios o manuales y la percepción auditiva o visual; la segunda, por los Sistemas de Pensamiento o Sistema Conceptual-Intencional, que se ocupa de producir pensamientos (categorizar el mundo o la mente ajena, planificar intencionalmente la acción, etc.). Ambos sistemas son los sistemas externos o limítrofes con la FL (pero «internos a la persona» según Chomsky 2002b, p. 26).

Desde esta perspectiva, la FL es una especie de puente entre los dos sistemas limítrofes, un mecanismo gracias al cual las representaciones generadas por esos dos sistemas se hacen accesibles entre sí de manera productiva. Dada tal arquitectura, surge la pregunta de cuál es la naturaleza de FL, siendo posibles dos respuestas: posición maximalista y posición minimalista (Longa y Lorenzo 2008). Según la primera, el lenguaje es una facultad específica de la mente/cerebro, caracterizada por imponer su propio formato representacional (la gramática) sobre los pensamientos para que estos puedan traducirse en instrucciones motoras. Por tanto, tal posición implica que la mente está dotada de un mecanismo gramatical autónomo, específico de dominio y muy articulado, que actúa como una «interlingua» entre dos sistemas que de otro modo serían mutuamente inaccesibles.

Por su parte, según la posición minimalista, el pensamiento se puede exteriorizar de manera directa, sin la necesidad de un sistema autónomo y autocontenido que permita ese trasvase, por lo que la mente no requiere ningún sistema gramatical específico de dominio. Según tal posición, los mecanismos de FL no son en esencia diferentes de los que componen los sistemas externos; por ello no es necesario asumir un formato específico (gramatical) para la FL.

Exceptuando el PM, el resto de modelos generativos, y dentro de ellos especialmente el Modelo GB, adoptaron inequívocamente la po- 
sición maximalista, al plantear una FL conformada por principios específicos de dominio (operantes solo en el ámbito gramatical), esto es, una FL claramente diferenciada en su naturaleza con respecto a los sistemas externos. Por su parte, el PM representa el paso a la posición minimalista, dado el rechazo a una consideración autónoma, autocontenida, de la FL. Los mecanismos de esta carecen de especificidad, siendo impuestos por los sistemas limítrofes, o derivados de condiciones de necesidad conceptual como las aludidas en el apartado anterior. En resumen, mientras la posición maximalista se basa en una gran cantidad de maquinaria específica a FL, no legible directamente por los sistemas externos, la minimalista se caracteriza por lo contrario, por eliminar toda la maquinaria específica de dominio. Las posiciones, pues, son contrarias y enfrentadas.

\section{AP. 2. ¿¿Cuál es la tesis central de ambos modelos?}

La respuesta del PM a esta cuestión se opone frontalmente, de nuevo, a la ofrecida unitariamente (de manera más o menos articulada) por los modelos previos. Estos, y GB como máximo representante, sostuvieron un rico estado inicial de conocimiento específicamente lingüístico, conformado por principios gramaticales que «do not arise in other cognitive domains» (Tracy 2002, p. 656). Eso significa que la tesis central de esos modelos, desde Chomsky 1965 a Chomsky 1981 y sucesivos trabajos en el marco de GB, fue la de la especificidad de FL, según la cual «el funcionamiento de la facultad lingüística se guía por principios específicos a ese dominio» (Chomsky 1980, p. 53). Por tanto, esos principios solo son operativos en un tipo de computaciones mentales, las que atañen a unidades lingüísticas.

Si GB plantea la tesis de especificidad del lenguaje, el PM defiende la tesis opuesta, de inespecificidad del lenguaje, que deriva de la Tesis Minimalista Fuerte, la cual asume, como posición de partida, el carácter perfecto u óptimo de la FL. Tal tesis sugiere que «FL no ofrece más maquinaria de la necesitada para satisfacer las exigencias mínimas de legibilidad y [...] funciona de la manera más simple posible» (Chomsky 2000a, p. 94). Estas palabras muestran que por buen diseño debe entenderse una arquitectura basada en su carácter inespecífico o no especial. Tal arquitectura está conformada por principios impuestos a la FL desde las interfaces con los dos módulos limítrofes o por principios que actúan de manera espontánea. Esto provoca la eliminación de todos los principios específicos de antaño. La mejor versión minimalista será la que postule una conexión más inmediata o directa (por ello óptima) entre los dos módulos externos, lo cual equivale a decir la que posea un mínimo de maquinaria gramatical, que estorbaría la inmediatez de esa 
conexión, pues debería ser traducida a términos de esos módulos. En resumen, la mejor teoría según el PM es la que evite en lo posible nociones y principios gramaticales autónomos, en los que se basaban precisamente los modelos previos. Estas palabras de Chomsky 2005, p. 9, son muy elocuentes: "we need no longer assume that the means of generating expressions are highly articulated and specific to language. We can seriously entertain the possibility that they might be reducible to language-independent principles».

El colofón es obvio: si el lenguaje es según GB altamente específico, y según el PM altamente inespecífico, no se aprecia en qué sentido las dos visiones pueden ser convergentes y complementarias. Más bien, ambas «embrace images of the FL not only conceptually in conflict but also mutually exclusive from an empirical point of view» (Longa y Lorenzo 2008, p. 554). La corrección de una implica la falsedad de la otra.

\section{AP.3. ¿Cuál es el estatus de los elementos gramaticales?}

Los elementos, niveles, operaciones y principios gramaticales han sido la clave de todos los modelos previos al PM. De hecho, se asumía que eran ellos los que ofrecían el conocimiento innato específico de dominio con que el aprendiz podría resolver el problema de la pobreza del estímulo y el correspondiente problema lógico de la adquisición del lenguaje. Dada, sin embargo, la tesis de inespecificidad elevada por el PM, en este modelo la situación cambia drásticamente. Como ya señalamos, si la Tesis Minimalista Fuerte se revelara acertada, FL tendría una estructura o diseño mínimo como puente entre los dos sistemas limítrofes que interconecta, donde por mínimo hay que entender carente de cualquier sustrato específicamente gramatical.

Así, los elementos gramaticales son considerados «imperfecciones» (Chomsky 2000a), pues al tener carácter autónomo, suponen elementos no directamente legibles (interpretables) por las interfaces con los módulos externos, con lo que entorpecen esa manera más sencilla de unión entre A-P y C-I. En resumen, la imperfección lo es porque carece de una justificación independiente más allá de la puramente gramatical. En tal sentido se deben entender las alusiones de Chomsky a que, según el Modelo GB, el lenguaje es altamente imperfecto; es así porque tal modelo se basaba precisamente en elementos puramente gramaticales.

\section{AP. 4. Por tanto, ¿qué papel se reserva a los elementos lingüisticos?}

El papel reservado a esos elementos (concordancia, proyecciones categoriales, caso, etc.) es fundamental en los modelos pre-minimalistas. 
En el PM sucede exactamente lo contrario: el papel reservado a ellos es cero, al menos como situación ideal de partida, o en su defecto el de ser un pequeño residuo en una estructura altamente inespecífica. Es difícil, otra vez, sostener la complementariedad de ambos enfoques atendiendo a tal aspecto.

AP. 5. ¿¿De dónde surge la motivación de la FL?

En GB (y en el resto de modelos generativos previos), la motivación de FL es interna, dado que es un sistema autocontenido y autosuficiente que impone sus características formales a los módulos limítrofes. Pero en el PM, FL pierde por completo su carácter autocontenido y autónomo, de modo que pasa a estar motivada por exigencias impuestas desde los sistemas limítrofes o por principios surgidos por necesidad conceptual. En ninguno de ambos casos se requieren instrucciones específicas o especiales a FL.

\section{AP. 6. ¿Qué papel tiene la modularidad en ambos modelos?}

GB caracterizó el lenguaje mediante una organización modular en dos sentidos diferentes: por un lado, FL es uno de los módulos o componentes especializados en los que se articula la mente, con un estatus similar a otros componentes mentales (cognición visual, teoría de la mente, control motor, etc.). Por otro, la noción de modularidad fue aplicada al seno de la propia FL. De ese modo, los principios y condiciones gramaticales se agrupaban en diferentes submódulos (X-barra, teoría temática, del caso, del ligamiento, etc.), relacionado cada uno de ellos con la sanción de aspectos diferentes de la gramaticalidad de las secuencias. En suma, GB asumió una modularidad externa (entre FL y otros sistemas) y otra interna (dentro de FL).

Es obvio que tal estado de cosas no podía mantenerse en el PM, dadas sus asunciones. Por un lado, la modularidad interna a FL es abandonada (cf. Hornstein 2009), por ser incompatible con los requisitos impuestos por la tesis de inespecificidad en dos sentidos: eliminación de todo tipo de elementos específicos y estructura mínima de FL. Esto es, una FL contemplada como altamente sensible a los requisitos funcionales dictados por los sistemas limítrofes, o a principios de necesidad conceptual, difícilmente podría seguir asumiendo una organización interna basada en divisiones estancas en el interior de la FL, que en conjunto convierten en muy tortuoso y opaco el contacto de los dos módulos limítrofes. 
Piénsese, por otro lado, que esa organización modular se vinculaba con diferentes niveles de representación, en especial dos niveles internos al lenguaje, Estructura-P y -S. Cada uno de esos niveles era el locus de aplicación de algunos de los submódulos en que se articulaba FL, para verificar que el proceso de generación se desarrollara exitosamente. Pero según el PM, los únicos niveles que se pueden mantener son aquellos en los que se entabla contacto entre FL y los sistemas limítrofes A-P y C-I. Los niveles de Estructura-P y -S no satisfacen tal criterio, pues son puramente internos a FL, y por ello, imperfecciones, al no tener ninguna justificación que no sea puramente gramatical. De ahí su eliminación (cf. Hornstein et al. 2005, cap. 2). Dada la concepción derivacional del modelo, no tiene sentido la noción de nivel de representación salvo en las interfaces de contacto con los dos módulos limítrofes. Las secuencias son legitimadas si son legibles por parte de ambos módulos, no habiendo ningún punto de control a lo largo del proceso (cf. Longa y Lorenzo 2001).

Por otro lado, en lo que respecta a la modularidad externa (FL como un módulo distinto de la mente), aunque no es eliminada, a la fuerza es relativizada (cf. Fox 2000, pp. 67-74). Esta relativización se sigue de los aspectos de arquitectura ya esbozados: FL ya no impone su formato específico a los dos módulos limítrofes, sino que se pliega a sus dictados, por lo cual es obvio que la FL pierde gran parte del carácter autocontenido y autónomo (nítidamente separado) que tuvo en la tradición generativa previa.

\section{AP.7. ¿Qué papel desempeña la Gramática Universal o genotipo lingüistico?}

La respuesta a esta pregunta es una de las que cuestionan más claramente la supuesta complementariedad y convergencia entre los enfoques comentados. Sin duda, la GU ha sido la noción central durante todo el devenir generativo (con independencia de su mayor o menor articulación en los diferentes modelos), pues reúne el conjunto de principios lingüísticos pre-especificados, objeto de conocimiento innato, con los que se caracteriza la aportación de la dote inicial al proceso de adquisición, sin la cual esta no es factible. Por tanto, esos principios conforman el genotipo lingüístico (Anderson y Lightfoot 2002, p. 22; Chomsky 1980, p. 75; Lightfoot 1982, p. 21; 1999, p. 52; 2006, p. 45). En consecuencia, la GU constituye un estado inicial genéticamente determinado (Chomsky 1980, p. 243) y específico para el lenguaje, definido así por Lightfoot 1982, p. 22: «The genotypical principles responsible for language acquisition can be viewed as a theory of grammar, sometimes called Universal Grammar. This represents the genetic equipment that makes language growth possible». 
Ya que «UG consists of the mechanisms specific to FL» (Chomsky 2007, p. 5), tales mecanismos son indeseables para el PM, pues su alta especificidad estorba la puesta en contacto más directa posible entre A-P y C-I. Tal noción no puede, pues, seguir manteniéndose en el PM, dada la primacía en él de la tesis de inespecificidad del lenguaje. Por tal razón, los principios gramaticales son eliminados, siendo sustituidos por:

a) principios impuestos por los módulos externos, y

b) condiciones de acción espontánea.

En consecuencia, el PM considera innecesario un blueprint específico para el lenguaje, lo que implica reducir drásticamente la carga genética requerida para su crecimiento. En resumen, la GU o «theory of the genetic endowment for language» (Chomsky 2009, p. 19) es reducida a un mínimo, o incluso eliminada por completo, como sugiere Chomsky 2004, p. 106, quien señala que la versión «extremely strong» de la Tesis Minimalista Fuerte implica que la GU («unexplained elements of $\mathrm{S}_{0}$ »), «is empty».

Nótese, pues, que el peso de la explicación se invierte por completo: la GU, verdadera noción clave en el generativismo durante treinta años, es contemplada por el PM como «the residue that constitutes FL once third factor properties of growth and development are extricated" (Chomsky 2007, p. 26; cf. también 2009, p. 25). Por ello, esa rica, específica y detallada estructura de conocimiento lingüístico inicial debe ser reemplazada, idealmente al menos, por otra en la que el referido estado inicial debe estar libre de cualquier atisbo gramatical (Lorenzo y Longa 2003). El acomodo de la GU en la agenda minimalista resulta problemático: el ideal del PM consiste en que cuanto menos relevante sea el factor genético, más perfecta será la FL. Chomsky 2009, p. 25, hace extensible estas consideraciones a la dimensión evolutiva del lenguaje: "the richer the residue, the harder it will be to account for the evolution of UG». Por otro lado, incluso aunque la investigación revele la necesidad de mantener un residuo de imperfección o versión mínima de la GU, «its residual character will impede us in thinking about it as a comprehensive program of development containing an articulated blueprint of any language» (Lorenzo y Longa 2009, p. 1306).

\section{AP. 8. ¿Son ambos modelos gencentristas?}

Como se desprende de la respuesta anterior, el generativismo siempre se caracterizó por asumir la necesidad de una carga genética para adquirir el lenguaje. Por ello afirma Jenkins 1979, p. 106, que la Gra- 
mática Generativa forma parte del «traditional study of the genetics of organisms». En esta misma línea, según Chomsky 1980, pp. 36-37, «el estudio de las propiedades del lenguaje genéticamente necesarias [...] se ocupa de determinar un aspecto de la genética humana, a saber, la naturaleza de la facultad lingüística». En resumen, los modelos preminimalistas fueron fuertemente gencentristas. Por el contrario, el PM rebaja drásticamente el papel del programa genético (cf. Longa 2006), enfatizando principios caracterizados por su inespecificidad. Esto implica que este modelo rechaza tajantemente el gencentrismo propio del Neo-Darwinismo, rechazando así dar un poder especial a los genes.

\section{AP.9. ¿Cuántos factores están implicados en el crecimiento del lenguaje?}

En GB, y en los modelos anteriores a este, solo se contemplaron dos factores implicados en el desarrollo del lenguaje: genes y entorno (experiencia a la que está sometido el niño). La adquisición del lenguaje es resultado de la interacción de ambos factores, y de ninguno más. El factor central es la GU, cuyos principios conforman la dote genética para el lenguaje, pero esos principios no están cerrados, sino que necesitan ser fijados en posiciones determinadas. La fijación de los principios en los parámetros acordes con el input recibido produce el estado estable de conocimiento de la lengua respectiva.

En el PM, sin embargo, se introduce por primera vez un tercer factor (Chomsky 2004, p. 105; 2005, p. 6; 2007, p. 3; 2009, p. 25; 2010, p. $51)$, que amplía el elenco de factores implicados en el desarrollo del lenguaje. A los ya conocidos de la dote genética (ahora reducida a un mínimo residual) y la experiencia, se añade un tercer factor, «Principles not specific to the faculty of language» (Chomsky 2005, p. 6). Sin embargo, este tercer factor, desconocido en la tradición previa, no es uno más añadido de manera cuantitativa, sino el factor decisivo, en el que el PM se centra a la hora de plantear la arquitectura de FL.

\section{AP. 10. ¿Qué papel desempeñan los principios independientes del lenguaje?}

En GB y modelos previos, los principios independientes del lenguaje no entraron de manera alguna en la agenda generativa, pues el énfasis se hacía en los principios de corte contrario, los específicos del lenguaje. Como señala Chomsky 1986, p. 11, «Mi idea es que los principios no se pueden generalizar, que en aspectos decisivos son específicos de la facultad lingüística». Por tanto, el papel reservado a los principios independientes del lenguaje se puede resumir en una palabra: nulo. 
En el PM, la respuesta es contraria: la explicación de la FL descansa, dada la tesis de inespecificidad del lenguaje, en este tipo de principios, que abarcan diferentes subtipos, como:

a) principios de análisis de datos y, sobre todo,

b) principios de arquitectura estructural y de computación eficiente (cf. Chomsky 2005, p. 6).

El PM no persigue una mera búsqueda de parsimonia, sino una verdadera agenda eliminativa de todo lo que tenga la impronta de específico al lenguaje, planteando en su lugar explicaciones muy genéricas. Por ejemplo, según el minimalismo los movimientos son los más cortos posibles no porque así lo dicte un módulo conformado por principios puramente gramaticales, como sucedía en GB, sino porque es la manera más eficiente, sencilla y económica de funcionamiento de cualquier sistema computacional. De ese modo, tal condición no necesita estar pre-especificada. No es una mera búsqueda de parsimonia la que origina el PM, frente a lo que sugieren los que enfatizan el minimalismo metodológico: realmente estamos ante una arquitectura y una reconsideración ontológica de FL completamente diferentes. En resumen, el PM implica «shifting the burden of explanation from the first factor, the genetic endowment, to the third factor, language-independent principles of data processing, structural architecture, and computational efficiency» (Chomsky 2005, p. 9).

Eso tiene otra interesante consecuencia, que acentúa aún más las diferencias con GB: las computaciones lingüísticas derivan de restricciones universales que no remiten a la evolución natural en sentido estricto (Uriagereka 1998), sino más bien a procesos usuales en las ciencias de la Complejidad: organización óptima de las formas y espacios, y simplicidad y generalidad de los procesos generativos. Como señala Chomsky 2009, p. 26, si la tesis minimalista fuera acertada, «language would be something like a snowflake, taking the form it does by virtue of natural law, in which case UG would be very limited». Esta predicción sería inesperada para GB.

\section{AP.11. ¿Qué significa una explicación basada en principios?}

A lo largo del desarrollo histórico del generativismo, una explicación basada en principios supuso una explicación centrada en condiciones específicas de dominio remitidas a la dote genética. Tales condiciones, ya desde Chomsky 1965, asegurarían la obtención de adecuación explicativa, «en la medida en que la teoría lingüística acierta a seleccionar una gramática descriptivamente adecuada sobre la base 
de datos lingüísticos primarios»(Chomsky 1965, p. 26). En resumen, esa explicación basada en principios se centraba en la formulación de un abundante aparato específico al lenguaje.

El PM plantea la óptica contraria: una explicación basada en principios (principled explanation; cf. Chomsky 2004; 2005; 2007, etc.) sobre el diseño de la FL supone ir «beyond explanatory adequacy» y solo se obtendrá con el abandono de la maquinaria específicamente gramatical, que GB tomaba como central, algo nítido en las siguientes citas (con nuestras aclaraciones entre corchetes):

Insofar as properties of L[anguage; VML y GL] can be accounted for in terms of IC [interface conditions; VML y GL] and general properties of computational efficiency and the like, they have a principled explanation (Chomsky 2004, p. 106).

[...] the goal is to determine just what aspects of the structure and use of language are specific to the language faculty, hence lacking principled explanation at this level (Chomsky 2004, p. 106).

We can regard an explanation of properties as principled insofar as it can be reduced to properties of the interface systems and general considerations of computational efficiency and the like (Chomsky 2005, p. 10).

En resumen, el objetivo del PM es indagar «to what extent its assumptions [de la GU; VML y GL] can be revised or eliminated in favour of principled explanation in terms of interface conditions and general principles» (Chomsky 2005, pp. 10-11). Por ello, todos aquellos principios o nociones puramente gramaticales, como la de rección, «seem to have no principled motivation» (Chomsky 2005, p. 11), dado que estorban la puesta en contacto más directa entre los dos módulos limítrofes: en otras palabras, son casos típicos de imperfecciones.

Así pues, el PM equipara una explicación basada en principios no con una naturaleza gramatical y específica de dominio, asunción central de todos los modelos previos, sino con la perspectiva contraria, caracterizada por su inespecificidad. Tal aspecto supone mucho más que una mera extensión de GB, basada en factores como simplicidad, economía, etc. Por ello, se antoja harto complicado entender en qué sentido puede haber convergencia, interacción y complementariedad entre ambas perspectivas.

\section{AP. 12. ¿Son modelos preformistas?}

La noción de preformismo está íntimamente vinculada con las de gencentrismo y programa genético. El preformismo moderno, resucitado por el Neo-Darwinismo desde la óptica de la información, supone 
considerar que los rasgos están pre-especificados (preformados) en un programa genético que contiene las instrucciones necesarias para el desarrollo de tales rasgos. Como escribe Mayr 1982, p. 106, el desarrollo del organismo «is controlled by something preformed, now recognized as the genetic program».

GB y los modelos previos fueron firmes defensores del preformismo, al considerar que los principios fundamentales del lenguaje estaban pre-especificados, necesitando solo ser activados por la experiencia lingüística. Por ello, y según tal esquema, es la información existente en los genes la que contiene los rasgos básicos del lenguaje. Esto es, la información lingüística está presente, pero no expresada hasta ser activada por la experiencia.

Muy diferente es lo que sucede con el PM. La rebaja o eliminación de la GU y, en consecuencia, de la noción de programa genético, provoca suprimir toda reminiscencia preformista, dada la supresión de cualquier tipo de elementos pre-especificados. FL se caracteriza, como hemos reiterado, por principios cuya justificación es externa a la propia FL: principios impuestos desde los dos módulos limítrofes, o principios que surgen merced a la auto-organización, derivada de la eficiencia computacional, para los que no es necesario imponer ninguna condición especial, que surgen por defecto porque son la solución más sencilla posible. Esto significa que los principios del PM no precisan ya estar impresos o preformados ni en los genes ni en el cerebro, sino que se siguen de manera espontánea en ausencia de cualquier otro criterio establecido al mismo efecto. En resumen, la FL no deriva de procesos genéticos, sino de procesos epigenéticos provocados por el contacto, durante el desarrollo del lenguaje, entre los dos sistemas limítrofes a FL (Lorenzo y Longa 2003, 2009).

\section{AP. 13. ¿Qué estatus recibe el lenguaje como rasgo biológico?}

La respuesta deriva del estatus gencentrista y preformista o no de ambos modelos. Según GB, el lenguaje es contemplado como un rasgo genotípico, al considerar que la fuente del conocimiento innato reside en el plano genético (genotipo lingüístico). Sin embargo, desde la óptica del PM, el asiento del lenguaje como rasgo innato no puede ser el mismo: FL no es tomada ya como un rasgo genotípico sino como un rasgo fenotípico. Lejos de responder a especificaciones codificadas en el genoma, el lenguaje es simplemente una propiedad determinada a aparecer de manera robusta y fiable durante el proceso de desarrollo de cualquier miembro de la especie (cf. Maclaurin 2002). Lo innato es la propiedad en sí, con independencia de los fundamentos de su desarrollo, que no tienen que ser genéticos. 
AP. 14. ¿Se identifican los planos genético e innato?

Dadas las premisas de GB, es obvia la férrea identificación de este modelo entre lo genético y lo innato. Esto ha sido recurrente en el NeoDarwinismo, que sostuvo la idea de que solo el nivel genético podría justificar el carácter innato de un rasgo. Tal visión, según han mostrado varias perspectivas desarrollistas, deja de lado diferentes mecanismos que no son genéticos pero se pueden considerar innatos (cf. Longa 2006). Desde tal perspectiva, un rasgo innato deriva de una interacción muy compleja entre recursos heterogéneos, algunos de ellos genéticos pero otros no genéticos. El desarrollo supone un proceso determinado de manera conjunta por múltiples causas, por lo que no es factible primar solo algunas de ellas (genéticas), ignorando el resto. Como escribe Wimsatt 1999, p. 160, «The equation of innate with genetic is ill founded -being genetic is not necessary nor sufficient for being innate. Equating innate with genetic is a kind of functional localization fallacyassuming that the function of a larger system or subsystem is realized completely in a part of that system».

El PM, aunque por otras razones (ya señaladas), asume tal perspectiva. Por supuesto, este modelo sigue considerando que el lenguaje es un rasgo innato, por lo que no rechaza ni relativiza el enfoque naturalista e internista sobre el lenguaje (Chomsky 1998b). Pero ya no considera necesaria una carga genética detallada para su surgimiento. El lenguaje como rasgo innato puede estar formado por variados recursos de desarrollo, como el tercer factor, que no son genéticos ni están específicamente codificados.

\section{Ap. 15. ¿Cómo se conceptualiza la adquisición?}

Según GB, la adquisición supone fijar los principios universales ofrecidos por la dote genética en posiciones determinadas, gracias a la experiencia. El peso del proceso es así desempeñado por el programa genético específico para el lenguaje. En el PM es obvio que la visión de la adquisición tiene que ser muy diferente: el proceso ya no consiste en desplegar capacidades específicas de dominio genéticamente determinadas, y que por ello preexisten, sino en considerar capacidades que no pertenecen en sentido estricto a FL y que no son específicamente lingüísticas, relacionadas con la conceptualización del entorno o la ejecución de planes motores por un lado, o con principios de optimización de las computaciones por otro (a pesar de la renuencia de los adquisicionistas generativos a considerar el PM; considérese el modelo propuesto en Lorenzo y Longa 2009). 
Dado que los fundamentos últimos de FL ya no son lingüísticos, es la interacción de los dos módulos limítrofes en el desarrollo la responsable de la aparición de FL. Por tanto, esta debe considerarse como una especie de efecto colateral del desarrollo de los sistemas externos, lo que supone que FL es un resultado inevitable a partir de condiciones iniciales no lingüísticas en lugar de ser el producto final de un estado inicial de conocimiento puramente lingüístico. En otras palabras, el proceso de adquisición supone emparejar unidades conceptualesintencionales y senso-motrices ofrecidas por los sistemas externos y combinadas merced a FL, procedimiento computacional regido por principios tan simples como inespecíficos (fusión binaria, proyección asimétrica, movimientos mínimos, etcétera).

Para resumir, mientras la concepción de la adquisición se basa en GB y en los modelos anteriores en elementos puramente lingüísticos, el PM plantea la hipótesis opuesta, consistente en evitar cualquier rastro de imperfección, centrando la explicación en una concepción inespecífica de la FL (cf. Lorenzo y Longa 2009, y las críticas de Lorenzo y Longa 2011 sobre la TPP como modelo de adquisición).

\section{AP. 16. ¿Es la adquisición el problema central?}

Desde los años sesenta, la adecuación explicativa ha sido el principal objetivo de los sucesivos modelos generativos. Como ya señalamos, tal nivel atañe directamente a la resolución del problema de la adquisición del lenguaje. Como señala Chomsky 1965, p. 27, «el problema de la justificación interna -de la adecuación explicativa- es, en esencia, el problema de construir una teoría de la adquisición del lenguaje, un dar cuenta de las habilidades innatas específicas que hacen posible este logro». Por tanto, la resolución de este nivel suponía formular una GU dotada de un rico conocimiento innato y específico de dominio con el que salvar la pobreza de los datos a los que accede el aprendiz. Así, múltiples generativistas han considerado al estudio de la adquisición como la esencia del enfoque:

The central problem is to characterize how children can master their native languages (Lightfoot 1982, p. 15).

A fundamental goal of linguistic theory is to explain how natural languages are acquired (Crain 1991, p. 597).

Sin embargo, las cuestiones vinculadas con la adquisición pierden esta centralidad con la llegada del PM (cf. Longa 1999b; Longa y Lorenzo 2001, cap. 4; Longa y Lorenzo 2008; y Lorenzo 2001, cap. 4). Como escribe Chomsky 2000a, pp. 76-77: 
Supongamos que una FL que satisface óptimamente las condiciones de legibilidad también satisface el resto de condiciones empíricas: adquisición, procesamiento, neurología, cambio lingüístico, etc. En ese caso el órgano del lenguaje es una solución perfecta a las especificaciones mínimas de diseño [...] En tanto que la tesis es verdadera, la información sobre otras cuestiones (conexiones sonido-significado, neurofisiología, etc.) puede ayudar en la práctica -incluso ser indispensable- para descubrir la naturaleza de FL y de sus estados. Pero en principio es irrelevante.

Esto es, según el PM, las teorías son evaluadas según su capacidad para explicar el ajuste entre FL y los módulos limítrofes con los que esta conecta, con lo cual la colocación en primer plano de las condiciones de legibilidad impuestas a FL desplaza a un segundo término la atención a la cuestión de la adquisición. De este modo, las propuestas concretas pasan a enjuiciarse en el PM en tanto que se funden en una base conceptual con un grado óptimo de ajuste de FL con los módulos externos y no según se funden en una base conceptual apta para dar cuenta del Problema de Platón. Por supuesto, eso no significa que la adquisición deje de tener relevancia; sucede, sin embargo, que ya no es la guía directriz del modelo.

\section{REFERENCIAS BIBLIOGRÁFICAS}

Anderson, S. y Lightfoot, D. (2002): The language organ. Linguistics as cognitive physiology, Cambridge, Cambridge University Press.

Boeckx, C. (2006a): Linguistic minimalism. Origins, concepts, methods, and aims, Nueva York, Oxford University Press.

Boeckx, C. (2006b): «Universals in a generative setting», en Mairal, R. y Gil, J. (eds.), Linguistic universals, Cambridge, Cambridge University Press, pp. 67-79.

CHERNIAK, C. (2005): «Innateness and brain-wiring optimization: Non-genomic nativism», en Zilhão, A. (ed.), Evolution, rationality, and cognition: A cognitive science for the twenty-first century, Nueva York, Routledge, pp. 103-112.

- (2009): «Brain wiring optimization and non-genomic nativism», en PiattelliPalmarini, M.; Uriagereka, J. y Salaburu, P. (eds.), Of minds \& language, Nueva York, Oxford University Press, pp. 108-119.

CHomsky, N. (1965): Aspects of the theory of syntax, Cambridge (MA), MIT Press (citado por Aspectos de la teoría de la sintaxis, Madrid, Aguilar, 1971).

- (1973): «Conditions on transformations», en Anderson, S. y Kiparsky, P. (eds), A Festschrift for Morris Halle, Nueva York, Holt, Rinehart \& Winston, pp. 232-286.

- (1980): Rules and representations, Nueva York, Columbia University Press (citado por Reglas y representaciones, Méjico, FCE, 1983).

- (1981): Lectures on government and binding, Dordrecht, Foris. 
- (1986): Knowledge of language, Nueva York, Praeger (citado por El conocimiento del lenguaje, Madrid, Alianza, 1989).

- (1993): «A minimalist program for linguistic theory», en Hale, K. y Keyser, S. J. (eds.), The view from building 20, Cambridge (MA), MIT Press, pp. 1-52.

- (1995a): The minimalist program, Cambridge (MA), MIT Press (citado por El programa minimalista, Madrid, Alianza, 1999).

- (1995b): «Bare phrase structure», en Webelhuth, G. (ed.), Government and binding theory and the minimalist program, Oxford, Blackwell, pp. 383-439.

- (1998a): "Some observations on economy in generative grammar», en Barbosa, P., Fox, D., Hagstrom, P., McGinnis, M. y Pesetsky, D. (eds.), Is the best good enough?, Cambridge (MA), MIT Press, pp. 115-127.

- (1998b): Una aproximación naturalista a la mente y al lenguaje, Barcelona, Prensa Ibérica.

- (2000a): «Minimalist inquiries: The framework», en Martin, R., Michaels, D. y Uriagereka, J. (eds.), Step by step, Cambridge (MA), MIT Press, pp. 89155 (citado por «Indagaciones minimalistas: el marco», Moenia 5, 1999, pp. 69-126).

- (2000b): The architecture of language, Oxford, Oxford University Press (citado por La arquitectura del lenguaje, Barcelona, Kairós, 2003).

- Belletti, A. y Rizzi, L. (eds.) (2002a): On nature and language, Cambridge, Cambridge University Press.

- (2002b): «Indagaciones minimalistas», en Catalá, N., Díez Calzada, J. A. y García-Albea, J. E. (eds.), El lenguaje y la mente humana, Barcelona, Ariel, pp. 21-47.

- (2004): «Beyond explanatory adequacy», en Belletti, A. (ed.), The cartography of syntactic structures, Vol. 3, Structures and beyond, Oxford, Oxford University Press, pp. 104-131.

- (2005): «Three factors in language design», Linguistic Inquiry 36, pp. 1-22.

- (2007): «Approaching UG from below», en Sauerland, U. y Gärtner, H.-M. (eds.), Interfaces + recursion = language? Chomsky's minimalism and the view from syntax-semantics, Berlín, de Gruyter, pp. 1-29.

- (2009): «Opening remarks», en Piattelli-Palmarini, M., Uriagereka, J. y Salaburu, P. (eds.), Of minds \& language, Nueva York, Oxford University Press, pp. 13-43.

- (2010): «Some simple evo devo theses: How true might they be for language», en Larson, R., Déprez, V. y Yamakido, H. (eds.), The evolution of language: Biolinguistic perspectives, Nueva York, Cambridge University Press, pp. $45-62$.

- y LASNIK, H. (1993): «The theory of principles and parameters», en Jacobs, J., von Stechow, A., Sternefeld, W. y Venneman, T. (eds.), Syntax: An international handbook of contemporary research, Berlín, de Gruyter, pp. 506-569.

CraIN, S. (1991): «Language acquisition in the absence of experience», $B e^{-}$ havioral and Brain Sciences 14, pp. 597-612.

Eguren, L. y Fernández Soriano, O. (2004): Introducción a una sintaxis minimista, Madrid, Gredos.

EISENBEIB, S. (2009): «Generative approaches to language learning», Linguistics 47 , pp. $273-310$. 
FoDOR, J. (1975): The language of thought, Nueva York, Thomas \& Crowell (citado por El lenguaje del pensamiento, Madrid, Alianza, 1984).

Fox, D. (2000): Economy and semantic interpretation, Cambridge (MA), MIT Press. Freidin, R. y VERGNAUd, J.-R. (2001): «Exquisite connections. Some remarks on the evolution of linguistic theory», Lingua 111, pp. 639-666.

FriedemanN, M.-A. y Rizzi, L. (2000): «The acquisition of syntax: introduction», en Friedemann, M.-A. y Rizzi, L. (eds.), The acquisition of syntax. Studies in comparative developmental linguistics, Harlow, Longman, pp. 1-25.

Hauser, M.; Chomsky, N. y Fitch, W. T. (2002): «The faculty of language: What is it, who has it, and how did it evolve?», Science 298, pp. 1569-1579.

Hornstein, N. (2001): Move!: A minimalist theory of construal, Malden (MA), Blackwell.

- (2009): A theory of syntax. Minimal operations and Universal Grammar, Nueva York, Cambridge University Press.

- Nunes, J. y Grohmann, K. (2005): Understanding minimalism, Nueva York, Cambridge University Press.

Hyams, N. (2002): «Clausal structure in child Greek: A reply to Varlokosta, Vainikka and Rohrbacher, and a reanalysis», The Linguistic Review 19, pp. 225-269.

JENkins, L. (1979): «The genetics of language», Linguistics and Philosophy 3, pp. 105-119.

KaYNE, R. S. (1994): The antisymmetry of sintax, Cambridge (MA), MIT Press.

KitAhARA, H. (2006): «Some notes on the minimalist program», en Boeckx, C. (ed.), Minimalist essays, Ámsterdam, Benjamins, pp. 3-15.

Lightfoоt, D. (1982): The language lottery: Toward a biology of grammars, Cambridge (MA), MIT Press.

- (1999): The development of language: Acquisition, change, and evolution, Oxford, Blackwell.

- (2006): How new languages emerge, Cambridge, Cambridge University Press.

LONGA, V. M. (1999a): La restricción de las gramáticas accesibles. La resolución del problema de la proyección en el dominio transformacional, Santiago de Compostela, Universidad.

- (1999b): «The status of transformations in the Minimalist Program and the logical problem of language acquisition: An apparent disagreement», Theoretical Linguistics 25, pp. 161-178.

- (2006): «No solo genes: el Programa Minimalista y la reformulación de la noción de innatismo», Ludus Vitalis 14, 26, pp. 141-170.

- (2008): «Una visión crítica sobre la noción de programa genético desde la lingüística: consecuencias para la conceptualización de la ontogenia del lenguaje», Verba 35, pp. 347-385.

- y Lorenzo, G. (2001): Derivación y representación: su alternancia cíclica en la teoría de la gramática, Noia, Toxosoutos.

- (2008): «What about a (really) minimalist theory of language acquisition?», Linguistics 46, pp. 541-570.

LORENZO, G. (2001): Comprender a Chomsky. Introducción y comentarios a la filosofía chomskyana sobre el lenguaje y la mente, Madrid, Antonio Machado Libros. 
- (2007a): «Lo que no hace falta aprender y lo que no se necesita saber. Nota crítica: Linguistic minimalism. Origins, concepts, methods, and aims, de Cedric Boeckx», Teorema 26, 2, pp. 141-148.

- (2007b): «El programa minimalista y el problema de Platón. Reflexiones sobre la aportación del minimalismo a los desafíos del desarrollo lingüístico». Estudios de Lingüistica. Universidad de Alicante 21, pp. 209-228.

- y LONGA, V. M. (2003): «Minimizing the genes for grammar. The minimalist program as a biological framework for the study of language», Lingua 113, pp. 643-657.

- (2009): «Beyond generative geneticism: Rethinking language acquisition from a developmentalist point of view», Lingua 119, pp. 1300-1315.

- (2011): «Developmental plasticity and linguistic variation», ms., Universidad de Oviedo y Universidad de Santiago.

Maclaurin, J. (2002): «The resurrection of innateness», The Monist 85, pp. 105-130.

MARTIN, R. y URiagereKa, J. (2000): «Introduction: Some possible foundations of the Minimalist Program», en Martin, R., Michaels, D. y Uriagereka, J. (eds.), Step by step, Cambridge (MA), MIT Press, pp. 1-29.

MAYR, E. (1982): The growth of biological thought. Diversity, evolution, and inheritance, Cambridge (MA), Harvard University Press.

RiemsDijK, H. VAN y Williams, E. (1986): Introduction to the theory of grammar, Cambridge (MA), MIT Press (citado por Introducción a la teoría de la gramáti$c a$, Madrid, Cátedra, 1990).

RizzI, L. (2000): «Remarks on early null subjects», en Friedemann, M.-A. y Rizzi, L. (eds.), The acquisition of syntax. Studies in comparative developmental linguistics, Harlow, Longman, pp. 269-292.

- (2004): «On the study of the language faculty: Results, developments, and perspectives», The Linguistic Review 21, pp. 323-344.

Ross, J. R. (1967): Constraints on variables in syntax, tesis doctoral, MIT.

Smith, N. (1999): Chomsky. Ideas and ideals, Cambridge, Cambridge University Press.

TRACY, R. (2002): «Growing (clausal) roots: All children start out (and many remain) multilingual», Linguistics 40, pp. 653-686.

URiagereKa, J. (1998): Rhyme and reason, Cambridge (MA), MIT Press.

VALOIS, D. (2000): «Introduction», Canadian Journal of Linguistics 45, pp. 1-5.

WiLson, E. (1998): Consilience. The unity of knowledge, Nueva York, Knopf (citado por Consilience. La unidad del conocimiento, Barcelona, Galaxia-Gutenberg, 1999).

Wimsatt, W. (1999): «Generativity, entrenchment, evolution, and innateness: Philosophy, evolutionary biology, and conceptual foundations of science», en Hardcastle, V. G. (ed.), Where biology meets psychology. Philosophical essays, Cambridge (MA), MIT Press, pp. 139-179. 